\title{
EVALUACIÓN DEL COMPORTAMIENTO PRODUCTIVO DE CUATRO HÍBRIDOS DE TOMATE, BAJO CONDICIONES PROTEGIDAS, UBICADO EN LA COMUNIDAD CHAGUITE GRANDE, DEPARTAMENTO DE JINOTEGA, NICARAGUA
}

\section{EVALUATION OF THE PRODUCTIVE BEHAVIOR OF FOUR TOMATO HYBRIDS, UNDER PROTECTED CONDITIONS, LOCATED IN THE CHAGUITE GRANDE COMMUNITY, DEPARTMENT OF JINOTEGA, NICARAGUA}

Rosa Isabel Lacayo Barrios ${ }^{1}{ }^{*}$ Lesly Junieth López Mercado ${ }^{*}$ Sury Aylem Zamora Mayorga ${ }^{3}$ Emilseth Carolina Padilla Duarte ${ }^{4}$

(Recibido/received: 02-agosto-2021; aceptado/accepted: 22-octubre-2021)

RESUMEN: En Nicaragua el cultivo del tomate (Solanum Lycopersicum L.) ocupa el primer lugar entre las hortalizas de mayor producción y consumo. En el presente estudio se evaluaron cuatro híbridos, tres procedentes de Corea del Sur y un material comercial, el experimento se estableció bajo sistema de agricultura protegida en una finca de un productor de la comunidad de chagüite grande, ubicada en el departamento de Jinotega en el ciclo de Primera 2019. El diseño experimental implementado fue Bloque Completo al Azar (BCA) con cuatro repeticiones y cuatro tratamientos. El rendimiento fue estimado mediante la metodología medias repetidas longitudinales y separaciones de medias a través de prueba de Tukey $(\alpha=0.05)$, de igual manera se realizaron correlaciones fenotípicas entre los componentes de rendimientos. Los análisis fueron realizados en el programa $R$ versión 4.0.2 (2020-06-22) a través de una de sus plataformas Rbio versión 141 (20-09-2020), el análisis físico y químico del suelo se realizaron seguimiento las metodologías de los laboratorios de las Universidad Nacional Agraria y la Universidad de Ingeniería. Según los resultados los híbridos que presentaron mejor comportamiento fueron Tisey y Miranda con rendimiento promedios de $109,728 \mathrm{~kg} \mathrm{ha}^{-1}$ y $104,695.9 \mathrm{~kg} \mathrm{ha}^{-1}$ respectivamente, de igual manera se muestran correlaciones positivas 0.97 para las variables número de frutos comerciales y el rendimiento, asimismo el suelo presento buenas propiedades física y química para buen desarrollo del cultivo.

PALABRAS CLAVE: Evaluación de Híbridos; Componentes de Rendimiento; Correlaciones Fenotípicas

\footnotetext{
${ }^{1}$ Ingeniero Agrícola, Universidad Nacional de Ingeniería, * Autor, email: rosaisabelacayo@gmail.com

2 Ingeniero Agrícola, Universidad Nacional de Ingeniería, * Autor, email: lilopezmerc@gmail.com

${ }^{3}$ Maestro en Ciencia con mención Mejoramiento Genético Vegetal, Investigadora en Hortalizas del INTA Nicaragua y Enlace del proyecto INTA KOPIA Tomate. Email: aylemayorga@inta.go.ni

${ }^{4}$ Maestro en Ciencia con mención en Sanidad Vegetal, Docente de la Universidad Nacional de Ingeniería UNIRUPAP, Managua, Nicaragua.
} 
ABSTRACT: In Nicaragua, the tomato crop (Solanum Lycopersicum L.) occupies the first place among the vegetables with the highest production and consumption. In the present study, four hybrids were evaluated, three from South Korea and a commercial variety, the experiment was established under a protected agriculture system in a farm of a producer from the community of Chagüite Grande, located in the department of Jinotega in the First cycle 2019. The experimental design implemented was Complete Random Block (BCA) with four repetitions and four treatments. The yield was estimated by means of the longitudinal repeated means methodology and mean separations through the Tukey test $(\alpha=0.05)$, in the same way phenotypic correlations were made between the yield components. The analyzes were carried out in the R program version 4.0.2 (2020-06-22) through one of its platforms Rbio version 141 (09-20-2020), the physical and chemical analysis of the soil was followed by the methodologies of the laboratories of the National Agrarian University and the University of Engineering. According to the results, the hybrids that presented the best performance were Tisey and Miranda with average yields of $109,728 \mathrm{~kg} \mathrm{ha}^{-1}$ and $104,695.9 \mathrm{~kg} \mathrm{ha}^{-1}$ respectively, in the same way positive correlations 0.97 are shown for the variables number of commercial fruits and the yield, also the soil presented good physical and chemical properties for good development of the crop.

KEYWORDS: Hybrid evaluation; yield components; phenotypic correlations

\section{INTRODUCCIÓN}

El cultivo del tomate (Solanum Lycopersicum L.) es una de las hortalizas de mayor importancia a nivel mundial, esto se debe a sus cualidades nutricionales altos contenido de antioxidantes y vitamina. Entre los países de América Latina de mayor producción se encuentran; Argentina, Brasil y Chile con producciones anuales de $150 \mathrm{t}$. países que contribuyen aproximadamente en el 70 \% de la producción mundial. (MIFIC, 2017).

Según (FAO, 2018), Centroamérica representa el 15.3\% de la producción mundial de tomate. Dentro de la región podemos destacar que el mayor productor de tomate es Guatemala con $331,338.00 \mathrm{~kg}$ ha $(58.56 \%$ de la producción regional), en segundo lugar, Nicaragua con $80,723.00 \mathrm{~kg}$ ha (14.27\%), en tercer lugar, Honduras con 76,567.00 kg ha (13.53\%), en cuarto lugar, Costa rica con $57,238.00 \mathrm{~kg}$ ha $(10.12 \%)$ y en último lugar. El salvador con 19,915.00 $\mathrm{kg}$ ha $(3.52 \%)$.

En Nicaragua, el cultivo de tomate constituye una actividad de gran importancia económica para pequeños y medianos productores. El consumo nacional de tomate se estima en 50 millones de kilogramos y para este mismo se cosechó 1,637 hectáreas. (MIFIC, 2017). Según (FAO, 2018) en Nicaragua se establecen 1,706 ha, con rendimientos de $41,963.9 \mathrm{~kg} \mathrm{ha}^{-1} \mathrm{lo}$ que conlleva a una producción final de $71,577 \mathrm{t}$, las zonas productoras de tomate de mayor importancia en Nicaragua se encuentran en los departamentos de Matagalpa, Jinotega y Estelí, particularmente en los Valles de Sébaco y Tomatoya.

La producción de tomate en Nicaragua se encuentra actualmente de acorde la demanda nacional, el comercio ha venido a aportar mejores precios a los productores y diferentes variedades para los consumidores. 
En el presente estudio se evaluaron cuatro hibrido de tomate bajo sistema de agricultura protegida, mediante la estimación de los componentes de rendimiento, correlaciones fenotípicas como también se determinaron las propiedades físico y químicas del suelo con el fin de identificar híbridos potenciales bajo un sistema de prácticas de manejo, con fin de ofertar nuevas tecnologías a los productores que les permita mejorar su rendimiento y optar a mejores mercados.

\section{METODOLOGÍA}

Ubicación del estudio: El experimento se realizó en la finca de un productor de la Comunidad de Chagüite, localizado entre las coordenadas $13^{\circ} 08^{\prime} 58^{\prime \prime}$ de latitud norte y $86^{\circ} 02^{\prime} 37^{\prime \prime}$ de longitud oeste, su altitud es de 970 msnm, departamento de Jinotega.

Diseño Experimental: Un diseño de bloque completo al azar (BCA) con cuatro repeticiones y cuatro tratamientos incluyendo el testigo, utilizando distancia de siembra de 1 metro entre surcos y 0,50 metro entre planta, para un total de 22 plantas por tratamiento y con un área total de $253 \mathrm{~m}^{2}$.

Tratamientos: En el estudio comprendió cuatro híbridos, tres procedente de Corea del Sur y un híbrido comercial procedente de Tailandia.

Tabla 1. Detalle del material genético utilizado en el estudio de evaluación del comportamiento productivo de cuatro híbridos de tomate bajo condiciones protegidas.

\begin{tabular}{ccccc} 
No. & Genotipos & $\begin{array}{c}\text { Composición } \\
\text { genética }\end{array}$ & $\begin{array}{c}\text { Habito de } \\
\text { crecimiento }\end{array}$ & Origen \\
\hline 1 & Tygo & Híbrido & Indeterminado & Corea del Sur \\
2 & Aarat & Híbrido & Indeterminado & Corea del Sur \\
3 & Miranda & Híbrido & Indeterminado & Corea del Sur \\
Testigo & Tisey & Híbrido & Semi-indeterminado & Tailandia \\
\hline Fuente: INTA-KOPIA & & &
\end{tabular}


Unidad experimental del estudio para estimación del potencial productivo y correlaciones: La muestra tomada en el presente estudio fueron 20 planta por repetición para un total de 60 planta por tratamiento, se realizó registro plantas individuales por tratamiento en siete cortes (cosechas), en todo el ciclo productivo. Variables Cuantitativas: Las variables medidas fueron: Número de frutos comerciales (NFC), Número de frutos no comerciales (NFNC), Peso de frutos comerciales y no comerciales (PFC y PFNC), las cuales fueron clasificadas siguiendo las normas (CODEX STAN 293-2007, 2007), clasificación por categoría (1 y 2) y los estándares de tamaños de fruto UUAA (2015) producción y norma para la exportación de tomate en invernadero.

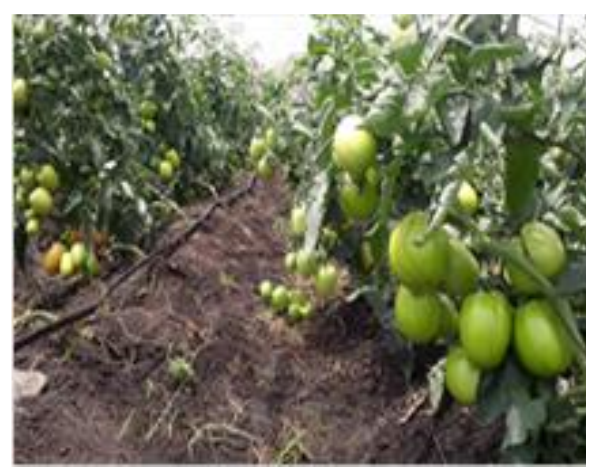

Figura 1: Experimento en etapa de producción, frutos del hibrido Miranda, comunidad Chagüite Grande Jinotega Ciclo primera

Los parámetros considerados para frutos comerciales fueron, enteros sanos, y exentos de podredumbre o deterioro, limpios, exentos de cualquier materia extraña visible; exenta de plagas, y daños causados por ellas, bien desarrollados con grado de madurez satisfactorio, pulpa suficientemente firme con peso mayor a $70 \mathrm{~g}$ y los frutos no comerciales inferiores a $70 \mathrm{~g}$.

Cantidad de muestras extraídas para el análisis de suelo:

Se extrajo una muestra de $1 \mathrm{~kg}$ del área de estudio, conformado por varias sub-muestras extraídas haciendo un corte en "V" utilizando un cilindro. Los análisis físicos y químicos se realizaron en los laboratorios de la Universidad Nacional Agraria (UNA) y la Universidad Nacional de Ingeniería (UNI). En análisis físico se consideró Capacidad de campo (C/c), Puntos de Marchitez Permanente (PmP), Densidad Aparente (Da), Densidad Real (Dr), Porosidad Total (Pt) Textura Mediante el método de Hidrómetro Bouyoucos, en el análisis químico se consideró Potencial de Hidrogeno, Conductividad Eléctrica (CE), Determinación de la Constante de la Celda en CE, Nitrógeno (N), Potasio(K), Fosforo Disponible (P) Material Orgánica (MO) utilizando el método de Walkley - Black.

Manejo del experimento: Se estableció bajo casa malla y se implementaron prácticas de manejo, tutoreo verticales

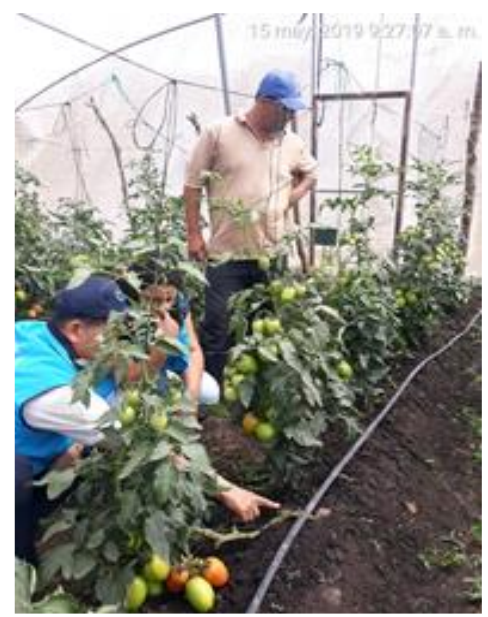

Figura 2: Experimento en etapa de producción, hibrido Tisey, comunidad Chagüite Grande Jinotega Ciclo primera 2019. de plantas individuales manejado a una guía, asimismo se realizaron podas de formación y aclareos para el buen desarrollo de las plantas, de igual manera se realizó monitoreo de plagas y enfermedades según la incidencia se realizaron aplicaciones de productos preventivos y selectivos. 
Procesamiento y análisis de datos:

Para la estimación del potencial productivo se realizó un análisis de varianza (ANDEVA) con separación de medias a través del test de Tukey al $(\alpha=0.05)$ de confianza, implementando un modelo mixto y un modelo aditivo lineal respectivamente, el programa utilizado fue $R$ versión 4.0.2 (2020-06-22) a través de una de sus plataformas Rbio versión 141 (20-09-2020).

Para la determinación de las asociaciones entre las variables que conforman los componentes de rendimiento se realizó un análisis de correlaciones fenotípicas utilizando $R$ versión 4.0.2 (2020-06-22).

\section{RESULTADOS Y DISCUSIÓN}

\section{- Estimación del potencial productivo de cuatro híbridos de tomate}

Las significancias obtenidas por cada variable evaluada, se determinó en base a los Criterios de Información Aikaike (AIC) y Criterios de Información Bayesianos (BIC), mediante el uso de matrices de covarianzas y estructuras del error, se identificó el modelo mejor se ajustado a la naturaleza de cada una de las mediciones (Tabla 2).

Tabla 2. $P$-valor de las variables de componentes de rendimiento.

\begin{tabular}{ccccccc} 
FV & NPFC & NPFNC & PTF & PPFC & PPFNC & RTOPT \\
\hline Bloque & 0.5406 & $<.0001^{* *}$ & $0.2697 \mathrm{Ns}$ & $<.0001^{* *}$ & 0.2855 & 0.0409 \\
& Ns & & & & Ns & Ns \\
Tratamiento & $<.0001^{* *}$ & $<.0001^{* *}$ & $<.0001^{* *}$ & $<.0001^{* *}$ & $<.0001^{* *}$ & $<.0001^{* *}$ \\
Cortes & $<.0001^{* *}$ & $<.0001^{* *}$ & $<.0001^{* *}$ & $<.0001^{* *}$ & $<.0001^{* *}$ & $<.0001^{* *}$ \\
(cosecha) & & & & & & \\
Tratamiento & $<.0001^{* *}$ & $<.0001^{* *}$ & $<.0001^{* *}$ & $1.00 \mathrm{E}-04$ & $<.0001^{* *}$ & $<.0001^{* *}$ \\
$\quad$ X Cortes & & & & $\mathrm{Ns}$ & & \\
$($ Cosecha) & & & & & & \\
\hline
\end{tabular}

FV: Fuente de variación, NPFC: Número promedio frutos comerciales, NPFNC: Número promedio frutos no comerciales, PTF: Peso total de frutos, PPFC: Peso promedio frutos comerciales, PPFNC: Peso promedio frutos no comerciales, Ns: No significativo, ${ }^{* *}$ : Mayormente significativo $(\alpha=0.5)$.

Según resultados del análisis estadístico mediante la selección del modelo más ajustado, se mostró que hubo diferencias significativas (<.0001) para las fuentes de variación: tratamiento (híbridos), cortes (cosechas) y la interacción de ambos para la mayoría de las variables de los componentes de rendimiento en excepto para la variable peso promedios de frutos comerciales.

Es decir que los híbridos difieren entre sí para todas las variables, asimismo hubo diferencias en las siete cosechas realizadas en todo el ciclo. 


\section{- Separaciones de Medias}

\section{- Rendimiento $\mathrm{kg} \cdot \mathrm{ha}^{-1}$}

Para la separación de medias por el Test de Rangos Múltiples de Tukey al $\alpha=0.05$ de confianza, realizada a través de un modelo aditivo, para la variable rendimiento se observan tres categorías definidas, en la categoría (a y ab) se ubicaron los híbridos Tisey, Miranda y Tygo con rendimiento $109,728,104,695.9$ y $89,375 \mathrm{~kg}^{-1} \mathrm{ha}^{-1}$ respectivamente y en la última categoría (b) se ubicó el hibrido Aarat con $83,743.25 \mathrm{~kg} \mathrm{ha}^{-1}$ (Ver tabla 3).

Tabla 3. Separaciones de media según Tukey $(\alpha=0.05)$ para las variables de rendimiento total kg. ha-1.

\begin{tabular}{ccc} 
Tratamiento & Rendimiento $\left(\mathrm{Kg}^{\mathrm{h}} \mathrm{ha}^{-1}\right)$ & Grupo \\
\hline Tisey & 109,728 & $\mathrm{a}$ \\
Miranda & $104,695.9$ & $\mathrm{ab}$ \\
Tygo & $89,375.96$ & $\mathrm{ab}$ \\
Aarat & $83,743.25$ & $\mathrm{~b}$ \\
\hline
\end{tabular}

Según (Olivas \& Salgado, 2013), describe rendimientos promedios de $94,500 \mathrm{~kg}$ ha-1 en una línea de tomate y rendimiento de $85,500 \mathrm{~kg}$ ha-1 con el híbrido shanty, bajo sistemas de agricultura protegida. Según el presente estudio, los híbridos evaluados superan los rendimientos en las categorías a y ab con $22 \%, 18 \%$ y $4 \%$ en comparación al estudio anteriormente descrito.

Por todo lado, (Andrades, Loaisiga , \& Freddy , 2015), en un proceso de evaluación de tres distancias de siembra se obtuvieron rendimiento $24971.56 \mathrm{~kg} \mathrm{ha}^{-1}$ bajo casa malla. Así mismo el INTA en el 2018, evaluó seis híbridos de los cuales solo Aarat y Shanty obtuvieron rendimiento promedio de $30,230 \mathrm{~kg} \mathrm{ha}^{-1}, 30,420 \mathrm{~kg} \mathrm{ha}^{-1}$. Según el presente estudio al evaluar los híbridos Tisey, Miranda, Tygo y Aarat, los cuatro superan los rendimientos en comparación a los resultados presentados en el año 2015 y 2018, esto debido a las nuevas implementaciones de tecnologías y manejo propuestas por INTA. Los rendimientos obtenidos fueron: Tisey con $109,728 \mathrm{~kg} \mathrm{ha}^{-1}$, Miranda con $104,695.9 \mathrm{~kg} \mathrm{ha}^{-1}$, seguido por Tygo con $89,375.96 \mathrm{~kg} \cdot \mathrm{ha}^{-1}$ y por último Aarat con $83,743.25 \mathrm{~kg} \mathrm{ha}^{-1}$.

\section{- Peso de frutos comerciales PPFC (kg.ha')}

Según el análisis por medio de Tukey $(\alpha=0.05)$, mostró que para la variable peso promedio de frutos comerciales agrupo a los híbridos de tomates en 2 categorías estadísticamente diferentes ( $\mathrm{a}$ y $\mathrm{b}$ ), siendo los híbridos Tisey, Miranda y Tygo que obtuvieron los mayores valores promedios diferenciándose de Tygo y Aarat (tabla 4).

Tabla 4. Separaciones de media según Tukey $(\alpha=0.05)$ para las variables pesos promedios de frutos comerciales. 


\begin{tabular}{ccc} 
Tratamiento & Medias (kg.ha-1) & Grupos \\
\hline Tisey & 100476.1 & $\mathrm{a}$ \\
Miranda & 97343.37 & $\mathrm{a}$ \\
Tygo & 70187.91 & $\mathrm{~b}$ \\
Aarat & 70156.83 & $\mathrm{~b}$
\end{tabular}

Lo que se afirma que los tratamientos Tisey y Miranda son híbridos que cumplen no solo con la norma de CODEX (CODEX STAN 293-2007, 2007) sino que también presenta pesos adecuados que permiten incursionar a mercados selectivos y de exportación.

- Correlaciones fenotípicas de variables de rendimiento entre los cuatro híbridos de tomate.

Según el análisis de las correlaciones fenotípica presentados en el mapa de calor se muestran correlaciones positivas y negativas entre las variables de rendimiento. Se identifica una correlación fenotípica positiva entre las variables Rto $\left(\mathrm{kg}^{\mathrm{h}} \mathrm{ha}^{-1}\right)$ y PPFC $(\mathrm{kg}$. ha-1) con 0.97, para los tratamientos: Tisey, Miranda, Tygo y Aarat, es decir que el mayor aporte al rendimiento se debió a los pesos promedios de Frutos comerciales.

Por otro lado, las variables PPFC (Peso Promedio de Frutos Comerciales) y PNFNC (Promedio Numero de Frutos No Comerciales) mostraron una correlación negativa de -0.86 así como también dicha variable con respecto al Rendimiento de - 0.76. De acuerdo a Ortega

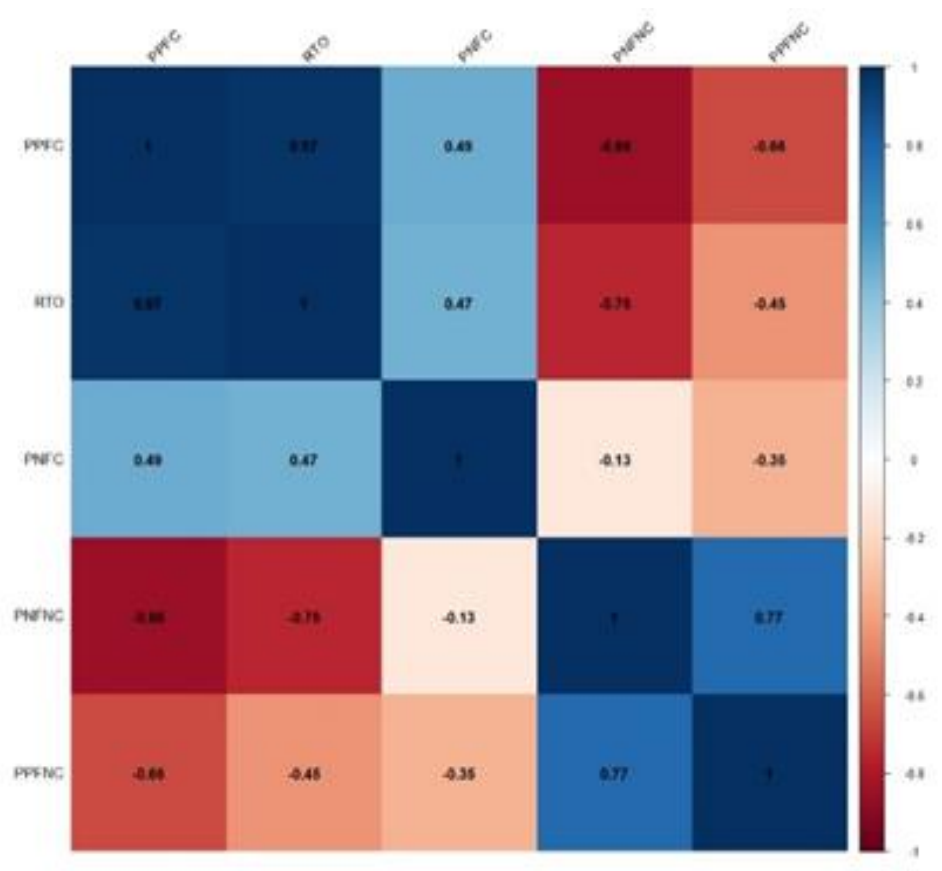

do una

Figura 3. Correlaciones fenotípicas para los Componentes de Rendimiento. Mapa de calor en $\mathrm{R}$ versión 4.0.3 (2020-10-10). 
- Análisis físico: Según los resultados, el suelo presento una textura arcillosa, un porcentaje de humedad del $30 \%$ considerándose un suelo con buena retención de agua para el establecimiento del cultivo.

Tabla 5. Detalles de los resultados del análisis físico

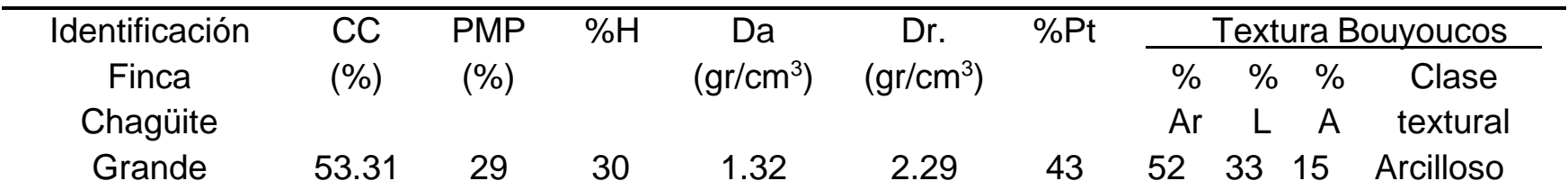

Nota: Cc: Capacidad campo, PMP: Punto de marchitez permanentes, \%H: Porcentaje de Humedad, Da. Densidad aparente, Pt: Porosidad total, Ar. Porcentaje de arcilla, L: Porcentaje de Limo, A: Porcentaje de arena.

Tabla 6. Detalle de los resultados del análisis químico

\begin{tabular}{ccccccc}
\hline Identificación & $\mathrm{pH}$ & $\begin{array}{c}\mathrm{MO} \\
\%\end{array}$ & $\begin{array}{c}\mathrm{CE} \\
(\mu \mathrm{s} / \mathrm{cm})\end{array}$ & $\begin{array}{c}\mathrm{N} \\
(\%)\end{array}$ & P-disp (ppm) & K-disp \\
$\begin{array}{c}\text { Finca Chagüite } \\
\text { Grande }\end{array}$ & 5.94 & 3.11 & 88.40 & 0.16 & 127.79 & $\begin{array}{c}(\mathrm{meq} / 100 \\
)\end{array}$ \\
\hline
\end{tabular}

Nota: $\mathrm{pH}$ : Potencial hidrogeno; MO: Materia orgánica; CE: Conductividad eléctrica; N: Nitrógeno, $\mathrm{P}$ disp: Fosforo; K-disp: Potasio.

Según (INETER, 2019) la temperatura media anual en Jinotega oscilo en $20.4{ }^{\circ} \mathrm{C}$ y precipitaciones de $1407 \mathrm{~mm}$.

Según la escala de (Lauritz Sorense, 2019) menciona que el pH adecuado para el cultivo de tomate es de 5.0 y 7.0 y en los análisis de suelo se obtuvo un pH de 5.94 estando este resultado en el rango óptimo para el establecimiento del cultivo de tomate.

Al hablar de fertilización de tomate, el (Centro de Investigaciones Agronomicas , 2016) indica que: La planta de tomate necesita absorber en promedio 2.5 a $3 \mathrm{~kg}$ de Nitrógeno, 0,2 a 0,4 kg de Fosforo y 2,6 a 3,6 kg de Potasio; Según los análisis realizados muestran que existe una deficiencia de nitrógeno con un $0.16 \mathrm{~N}$, fósforo con $127.79 \mathrm{P}(0.12 \mathrm{~kg})$ y de potasio con $0.92 \mathrm{Kg}$, por lo que se efectúa una programación de fertilización para proveer al cultivo los nutrientes que están faltando y que permiten la limitación y producción exitosa del cultivo de Tomate.

Como parte del manejo adecuado del suelo se realizaron diferentes fertilizaciones edáficas, en el transcurso del crecimiento vegetativo del cultivo tales como: Al momento del trasplante se aplicó 18-46-00 (Fosfato diamónico) con dosis de $325 \mathrm{~kg} \mathrm{ha}^{-1}$, posteriormente a los 20 días después del trasplante (ddt) se aplicó 46-0-0 (Urea) con dosis de $130 \mathrm{~kg} \mathrm{ha}^{-1}$, luego a los 40 ddt se aplicó 46-0-0 (Urea -130 $\mathrm{kg} \mathrm{ha}^{-1}$ ) y 0-0-60 (Muriato de potasio - $65 \mathrm{~kg} \mathrm{ha}^{-1}$ ), la última aplicación se efectuó a los 55 ddt con 46-0-0 (Urea - $130 \mathrm{~kg} \mathrm{ha}^{-1}$ ) y 0-0-60 (Muriato de potasio - $\left.65 \mathrm{~kg} \mathrm{ha}^{-1}\right)$. Asimismo, se realizaron aplicaciones fertilizantes foliares multiminerales Bayfolan® tres veces por semana ( $2 \mathrm{ml}$ por litro de agua). 


\section{CONCLUSIONES}

- Los híbridos de mayor rendimiento fueron Tisey, Miranda y Tygo con 109,728 kg ha-1, $104,695.9 \mathrm{~kg} \mathrm{ha}^{-1}$ y $89,375.96 \mathrm{~kg} \mathrm{ha}^{-1}$ respectivamente.

- Según los resultados hubieron correlaciones positivas altas Rto $\left(\mathrm{kg} \cdot \mathrm{ha}^{-1}\right)$ y PPFC $\left(\mathrm{kg} . \mathrm{ha}^{-1}\right)$ con 0.97 .

- Según la separación de medias los híbridos Tisey y Miranda el mayor aporte de al rendimiento fue de frutos comerciales.

- Según el análisis físico y químico la textura de suelo es Arcillosa, con pH de 5.94, proporcionando una óptima retención de humedad de 30\%, presentando poca aireación debido a su bajo porcentaje de poros $43 \%$, el contenido de materia orgánica fue de $3.11 \%$ clasificándola como media, indicando que el suelo es rico en nutrientes, considerándose apto para el cultivo de tomate.

\section{AGRADECIMIENTO}

Los autores expresan su gratitud al Instituto Nicaragüense de Tecnología Agropecuaria (INTA) y al Proyecto INTA KOPIA Tomate y la asesoría de la Msc. Sury Zamora, por darnos la oportunidad de ser parte de presente investigación, al mismo tiempo agradecer a la Universidad Nacional de Ingeniería por su asesoría al concluir este proyecto de investigación.

\section{BIBLIOGRAFIA}

Andrades, C., Loaisiga , J., \& Freddy , A. (2015). Evaluacion del crecimioento y rendimiento del cultivo de tomate (Lycopersicum sculemtum Mill)variedad shanty en tres distancias de siembra, en condiciones de casa malla,UNA,Managua,2015. Managua.

Centro de Investigaciones Agronomicas , U. (2016). Fertilizacion de Tomate. Obtenido de https://www.cia.ucr.ac.cr/pdf/Memorias/FERTILIZACION\%2520TOMATE\%25202016 .pdf\&ved=2ahUKEwj9xd7Y3JB0AhXXTTABHUdaAU4QFnoECAoQAQ\&usg=A0vVa w1zoeFT-ubUDPYSmPCKIf_

CODEX STAN 293-2007. (2007). Norma para el Tomate (CODEX STAN 293-2007).

FAO, O. A. (2018). FAO. Obtenido de http://www.fao.org/3/s8630s/s8630s08.htm

INETER, I. E. (2019). Boletin Climatico de la IIDecena de Febrero 2019. Obtenido de https://www.ineter.gob.ni/boletines/Boletin\%2520climatico/decenal/2019/febrero/Bolcl imatlldecFebrero2019.pdf\&ved=2ahUKEwjL3Jqx0Jb0AhXBQTABHbZRD34QfnoECA MQAQ\&usg=AOvVaw2siC-sWPHAqQ1vRRH-K8je

Lauritz Sorense, P. (Enero de 2019). Prociencia Informa. Obtenido de htpps://www.fondecyt.go.pe/fondecyt-informa/s-p-l-sorense-el-bioquimico-danescreador-del-potencial-de-hidrogeno-ph 
MIFIC, M. C. (2017). FICHA DEL TOMATE. Obtenido de https://cenida.una.edu.ni/relectronicos/RENE71N583ft.pdf

Olivas , L. A., \& Salgado, L. R. (2013). Evaluación de rendimiento y comportamiento agronómico de siete genotipos de tomate (Lycopersicum esculentum, Mill.) bajo sistema de casa malla en el centro experimental Las Mercedes Universidad Nacional Agraria.

\section{SEMBLANZA DE LOS AUTORES}

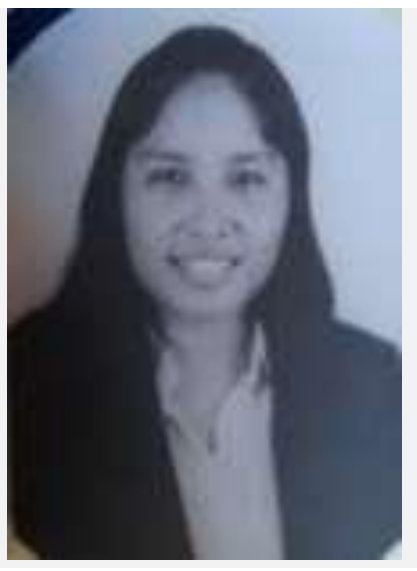

Rosa Isabel Lacayo Barrios: Ingeniera Agrícola, de la Universidad Nacional de Ingeniería, Facultad de la Tecnología de la construcción.

Email: rosalacayo18@gmail.com

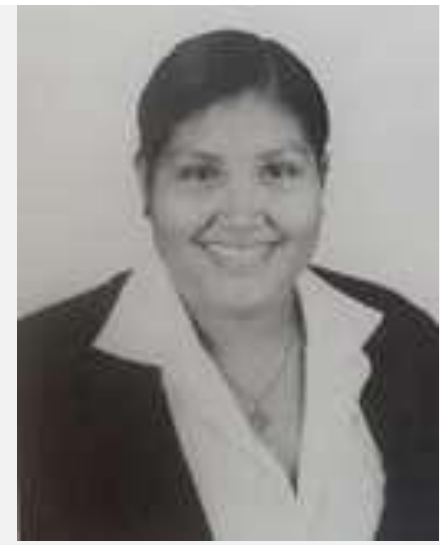

Lesly Junieth López Mercado: Ingeniera Agrícola, de la Universidad Nacional de Ingeniería, de la Facultad de Tecnología de la Construcción, innovador en formación. E-mail: ljlopezmer@gmail.com. 


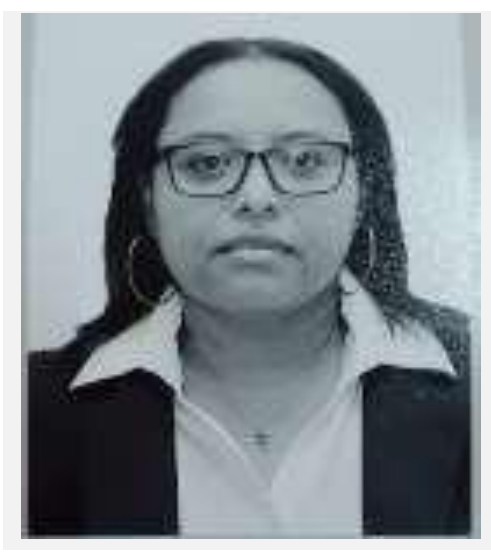

Emilseth Carolina Padilla Duarte: Ingeniera Agrícola, de la Universidad Nacional de Ingeniería, con Maestría en Sanidad Vegetal, Docente universitaria de la Facultad de la Tecnología de la construcción por más de 11 años.

Email: emilsethp@gyahoo.es

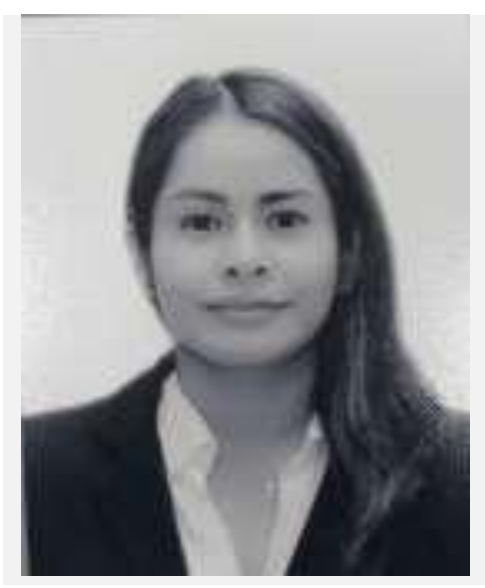

Sury Aylem Zamora Mayorga: Ingeniera en Sanidad Vegetal, de la Universidad Nacional Agraria, con Maestría en Mejoramiento Genético Vegetal, Investigadora en hortalizas del INTA Nicaragua y Enlace del proyecto INTA KOPIA Tomate. Email: aylemayorga@yahoo.com 